REFERENCES:

[1] Kyeong Min Son, Seong Hun Kang, Young II Seo, Hyun Ah Kim. Association of body composition with disease activity and disability in rheumatoid arthritis. Korean J Intern Med. 2021 Jan;36(1):214-222.

Disclosure of Interests: None declared

DOI: 10.1136/annrheumdis-2021-eular.3337

\section{POS0571 NON-OBESE RHEUMATOID ARTHRITIS PATIENTS WITH LOW LOW-DENSITY LIPOPROTEIN HAVE GREATER CORONARY ATHEROSCLEROSIS BURDEN, PLAQUE PROGRESSION AND CARDIOVASCULAR EVENT RISK}

G. Karpouzas ${ }^{1}$, S. Ormseth ${ }^{1}$, E. Hernandez ${ }^{1}$, M. Budoff ${ }^{2}{ }^{1}$ The Lundquist Institute, Rheumatology, Torrance, United States of America; ${ }^{2}$ The Lundquist Institute, Cardiology, Torrance, United States of America

Background: Rheumatoid arthritis (RA) patients with low body weight incur higher mortality than obese patients. Paradoxically, RA patients in the lowest low-density lipoprotein group ( $\mathrm{LDL}<70 \mathrm{mg} / \mathrm{dl}$ ) may also experience higher cardiovascular disease (CVD) risk.

Objectives: We here explored whether abdominal obesity (waist-to-height ratio $>0.58$ in females and $>0.63$ in males) might moderate the effect of low LDL $(<70 \mathrm{mg} / \mathrm{dl})$ on coronary atherosclerosis burden, progression and long-term CVD risk in RA.

Methods: One hundred fifty patients without symptoms or diagnosis of CVD underwent coronary computed tomography angiography. Plaque progression was evaluated $6.9 \pm 0.3$ years later in 101 patients. Coronary artery calcium, number of segments with plaque (segment involvement score), and extensive ( $>4$ segments with plaque) or obstructive disease ( $>50 \%$ stenosis) were assessed. CVD events were prospectively recorded, including cardiac death, myocardial infarction, unstable angina, revascularization, stroke, claudication, and heart failure hospitalization over $6.0 \pm 2.4$ years of follow-up. Lipoprotein classes were directly measured. Oxidized LDL (oxLDL) was assessed with monoclonal antibody E06. Adjusted robust linear regression evaluated interactions between abdominal obesity and LDL groups on plaque outcomes. Per segment, adjusted robust logistic regression models explored obesity $\mathrm{x}$ LDL group interactions on new plaque formation and stenotic progression of prevalent plaques. Robust Cox regression models stratified by abdominal obesity evaluated the effect of LDL group ( $<70 \mathrm{vs.}>70 \mathrm{mg} / \mathrm{dl}$ ) on CVD events.

Results: Non-obese patients with low LDL had the highest plaque burden (Figure $1 \mathrm{~A}$, all $\mathrm{p}<0.02$ ). Obesity moderated the effect of $\mathrm{LDL}$ on likelihood of extensive/obstructive disease $(P$ for interaction $=0.061)$; specifically, $\mathrm{LDL}<70$ associated with an increased likelihood of extensive/obstructive plaque in non-obese (adjusted OR 4.75 [95\% Cl 1.18-19.07], $P=0.028$ ) but not obese patients (adjusted OR $1.55[95 \% \mathrm{Cl} 0.39-6.08], P=0.532$ ). No differences in disease activity or inflammatory markers were seen across groups. Compared to LDL $>70 \mathrm{mg} / \mathrm{dl}$, low LDL predicted an increased likelihood of high oxLDL (>median) in non-obese patients (adjusted OR 5.10 [95\% Cl 1.46-17.75], $P=0.011$ ) but not obese patients (adjusted OR 0.50 [95\% Cl 0.11-2.21], $P=0.36$, Figure 1B). Obesity further moderated the effect of LDL on likelihood of future development of plaque ( $P$ for interaction $=0.002)$ and increased stenotic severity of existing plaques $(P$ for interaction $=0.040$ ); in non-obese patients, low LDL associated with a greater likelihood of new plaque forming in segments without baseline plaque (adjusted OR 4.68 [95\% Cl 2.26-9.66]) and worsening stenotic severity in segments with prevalent plaque (OR 5.35 [95\% $\mathrm{Cl} 1.62-17.67])$. This was not observed in obese patients (Figure 1C). Notably, in non-obese patients, low LDL associated with higher CVD event risk compared to those with LDL>70 mg/dl (HR 7.94 [95\% $\mathrm{Cl} 1.52-41.36], P=0.015)$. This was not the case in obese patients (HR 0.32 [95\% Cl 0.04-2.40], $P=0.27$, Figure 1D).

Conclusion: In non-obese RA patients, $\mathrm{LDL}<70 \mathrm{mg} / \mathrm{dl}$ may reflect higher $\mathrm{LDL}$ oxidation and was associated with higher baseline coronary atherosclerosis burden, new plaque formation, stenotic plaque progression and greater CVD risk than LDL>70 mg/dl.

Disclosure of Interests: George Karpouzas Speakers bureau: Sanofi/ Genzyme/ Regeneron, Consultant of: Sanofi/ Genzyme/ Regeneron, Grant/research support from: Pfizer, Sarah Ormseth: None declared, Elizabeth Hernandez: None declared, Matthew Budoff Consultant of: Pfizer

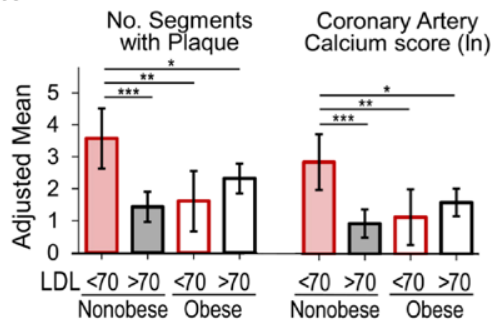

C

\begin{tabular}{|c|c|c|c|}
\hline Outcome & Adjusted & d OR $(95 \% \mathrm{Cl})$ & $P$ value \\
\hline \multicolumn{4}{|c|}{ Increased stenosis } \\
\hline Obese & & $1.1(0.1-15.2)$ & 0.958 \\
\hline Non-obese & $\rightarrow$ & $5.4(1.6-0.17 .7)$ & 0.006 \\
\hline \multicolumn{4}{|l|}{ New plaque } \\
\hline Obese & $\rightarrow$ & $0.7(0.2-2.5)$ & 0.562 \\
\hline Non-obese & $\rightarrow$ & $4.7(2.3-9.7)$ & $<0.001$ \\
\hline \multicolumn{4}{|c|}{$\begin{array}{llllll}0.01 & 0.1 & 1.0 & 10 & 100\end{array}$} \\
\hline $\begin{array}{l}\text { Highe } \\
\text { LDL>> }\end{array}$ & $\begin{array}{l}\text { rfor Higher } \\
70 \quad \text { LDL }\end{array}$ & & \\
\hline
\end{tabular}

Figure 1 (A) Mean number of coronary segments plaque and log-transformed coronary artery calcium score across LDL/obesity groups, adjusted for a an gender, diabetes, and statin use. (B) Percent patients with elevated (>median) oxidized LDL (OxLDL) across LDL/obesity groups, (B) Pusted for age adjusted for age, gender, hypertension, diabetes, and statin use. (C) Moderating effects of obesity on the association between low LDL and worsening stenosis of existing plaques and new plaque formation in coronary segments without baseline plaque, adjusted for age, time between (D) The in dul

$* p<0.050,{ }^{* *} p<0.010,{ }^{* * *} p<0.001$.

DOI: 10.1136/annrheumdis-2021-eular.3419

\section{POS0572 THE ASSOCIATED FACTORS OF ULTRASONOGRAPHIC TENOSYNOVITIS IN PATIENTS WITH RHEUMATOID ARTHRITIS IN REMISSION}

R. Fakhfakh ${ }^{1}$, N. El Amri ${ }^{1}$, K. Baccouche ${ }^{1}$, H. Zeglaoui ${ }^{1}$, E. Bouajina ${ }^{1} .{ }^{1}$ Farhat Hached Hospital, Rheumatology, Sousse, Tunisia

Background: Tenosynovitis is a common manifestation of rheumatoid arthritis (RA), but, is difficult to assess appropriately by clinical examination. In clinical remission, musculoskeletal ultrasound (MSUS) showed a predictive value of tenosynovitis for flares and joint destruction in RA (1).

Objectives: To assess the prevalence, the characteristics, and the factors associated with tenosynovitis in ultrasound examination of patients with RA in clinical remission.

Methods: A Cross-sectional study including patients with RA in clinical remission (DAS28 ESR $\leq 2.6$ ) for at least 6 months. MSUS of 20 tendons (in wrists, hands and ankles) was performed in mode $\mathrm{B}$ and power Doppler (PD). Tenosynovitis was defined and scored according to the Outcome Measures in Rheumatology Clinical Trials (OMERACT). A total score for the tenosynovitis in B-mode (TBI) and PD (TDI) was calculated by summing the grades for each patient. SDAI and CDAI were calculated. Functional disability was assessed by the Health Assessment Questionnaire (HAQ). For the radiographic evaluations, we used the Van der Heijde score.

Results: Thirty-seven patients were enrolled, the mean age was 54.2 years \pm 12.7 years and the sex ratio was 0.37 . The mean disease duration was 8.1 years \pm 5.1 . The mean remission duration was 36.5 months \pm 32.7 . The prevalence of tenosynovitis was $40.5 \%$ in B-mode and $13.5 \%$ for PD. The most affected tendons were: extensor carpi ulnaris (B-mode: $21.6 \%$, PD: $8.1 \%$ ), tibialis posterior (B-mode: 10\%, PD: $2.7 \%$ ), peroneal (B-mode: $8.1 \%$, PD: $2.7 \%$ ), flexor digitorum superficialis and profundus (B-mode: $5.4 \%, \mathrm{PD}: 0 \%$ ) and extensor digitorum (B-mode: 2.7\%, PD: 2.7\%). Tenosynovitis was most frequently grade1 in B-mode $(32.4 \%)$ and grade 2 in PD $(10.8 \%)$. Grade 3 was identified only in B-mode in the peroneal tendons. The mean of TBI was $7.1 \pm 5.3$ and the mean of TDI was 2.2. \pm 3.1 . We found a significant association between remission duration and $P D$ tenosynovitis (mean: 16.2 vs 39.7 months, $p=0.05$ ). Tenosynovitis, in $B$-mode and PD, weren't associated with the swollen joints, the tender joints, the patient 
global health assessment, the $\mathrm{HAQ}$, erythrocyte sedimentation rate, C-reactive protein, rheumatoid factor and anti-cyclic citrullinated peptide, DAS28, CDAI, SDAI, biologic treatment and radiographic structural damage. Multivariate logistic regression analysis didn't show any association.

Conclusion: Ultrasound tenosynovitis was commonly found in RA in remission and the extensor carpi ulnaris tendon was most involved. A shorter duration of remission was associated with PD tenosynovitis in univariate analysis. The MSUS assessment of tendons can be an additional feasible method to assess clinical remission. REFERENCES:

[1] Janta I, Stanciu D, Hinojosa M, Nieto-González JC, Valor L, Bello N, et al. Structural damage in rheumatoid arthritis: comparison between tendon damage evaluated by ultrasound and radiographic damage. Rheumatology (Oxford). 2016;55(6):1042-6.

Disclosure of Interests: None declared

DOI: 10.1136/annrheumdis-2021-eular.3511

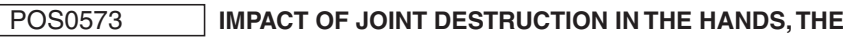 WRISTS AND THE ANKLES ON THE ULTRASOUND ASSESSMENT AND THE FUNCTIONAL OUTCOMES IN RHEUMATOID ARTHRITIS IN REMISSION}

R. Fakhfakh ${ }^{1}$, N. El Amri ${ }^{1}$, K. Baccouche ${ }^{1}$, H. Zeglaoui ${ }^{1}$, E. Bouajina ${ }^{1} .{ }^{1}$ Farhat Hached Hospital, Rheumatology, Sousse, Tunisia

Background: Joint destruction is a strong predictive factor for residual synovitis among rheumatoid arthritis (RA) patients in clinical remission. Both of them were associated with functional impairment.

Objectives: To assess the ultrasound findings and functional outcomes of RA patients in remission according to the site of joint destruction.

Methods: A Cross-sectional study including RA patients in remission DAS28 ESR $\leq 2.6$ for at least 6 months. A B-mode and power doppler (PD) ultrasound of 42 joints was performed. Synovitis was defined and scored using the combined OMERACT-PDUS (gray scale and power doppler (PD)) scoring system graded from 0 to 3 . The health assessment questionnaire (HAQ) and the radiological Sharp score of the wrists, hands and feet were calculated.

Results: Thirty-seven patients were included. The sex ratio was 0.37 and the mean age was 54.2 years \pm 12.7 . The mean disease duration was 8.1 years \pm 5.1 . The mean remission duration was 36.5 months \pm 32.7 . The mean DAS28vs was $2.1 \pm 0.5$. Rheumatoid factor and anti-citrullinated peptide antibodies were found in $62 \%$ and $75 \%$ of patients, respectively. The mean HAQ was $0.35 \pm 0.38$. Bone erosion was found in $81 \%$ of patients. In patients with hands and feet erosions (54\%), synovitis was found in $90 \%$ of cases associated with PD in $70 \%$ of cases. The mean total score of synovitis was $7.8 \pm 5.4$. The mean HAQ was $0.37 \pm 0.44$. In patients with only erosions in the hands or wrists (18.9\%), synovitis was found in $100 \%$ of cases associated with PD in $57 \%$ of cases. The mean total score of synovitis was $4.5 \pm 4.7$. The mean HAQ was $0.48 \pm 0.34$. In patients with only feet erosions $(8.1 \%)$, synovitis was found in $100 \%$ of cases associated with PD in $66.7 \%$ of cases. The mean total score of synovitis was $4.6 \pm 3.5$. The mean HAQ was $0.31 \pm 0.26$. RA was not erosive in $18.9 \%$ of patients. In these patients, synovitis was found in $100 \%$ of cases associated with PD in $28.6 \%$ of cases. The mean total score of synovitis was $5.6 \pm 4$.3. The mean HAQ was $0.21 \pm 0.23$. There is no significant difference between these groups $(\mathrm{p}>0.05)$.

Conclusion: In RA in remission, PD synovitis and functional incapacity were less frequent in the absence of erosion. PD synovitis was more frequently found in patients with erosions, especially, in the feet. The HAQ was higher in patients with hands erosions.

\section{REFERENCES}

[1] Hamamoto Y, Ito H, Furu M, Hashimoto M, Fujii T, Ishikawa M, et al. Serological and Progression Differences of Joint Destruction in the Wrist and the Feet in Rheumatoid Arthritis - A Cross-Sectional Cohort Study. PLoS One. 2015;10(8):e0136611.

Disclosure of Interests: None declared

DOI: 10.1136/annrheumdis-2021-eular.3601

\section{POS0574 $\quad$ RELATIONSHIP BETWEEN INVOLVED JOINT FOR REGION AND MODIFIED HEALTH ASSESSMENT QUESTIONNAIRE SCORE IN JAPANESE PATIENT WITH RHEUMATOID ARTHRITIS}

I. Yoshii . ${ }^{1}$ Yoshii Hopital, Rheumatology and Musculoskeletal Medicine, Shimanto City, Japan

Objectives: Rheumatoid arthritis (RA) is a chronic inflammatory disease that involves various joints in whole body. For evaluation of daily life activities (ADL), modified Health Assessment Questionnaire (mHAQ) is usually used. This index configures eight ADL functions these are separated by predominant extremities. This study aimed to evaluate how involved joint affect ADL predominantly in real world setting. Methods: A total of 24,450 times of consultation with RA patient were visited in the institute. Here, patient with RA was interviewed every another visit, and involved joint in whole body, pain score with visual analog scale (PS-VAS), and $\mathrm{mHAQ}$ were recorded. Involved joints were divided by four regions in accordance with joint size and part; small joint in upper extremities (US), large joint in upper extremities (UL) small joint in lower extremities (LS), and large joint in lower extremities (LL). mHAQ was also separately evaluated in accordance with predominant regions; uppe extremities predominant $\mathrm{mHAQ}$ ( $\mathrm{mHAQ} U \mathrm{UE})$, and lower extremities predominant $\mathrm{mHAQ}$ (mHAQ_LE). Adding to these parameters, as an index for disease activ ity monitoring, components of the simplified disease activity index score (SDAI) was also recorded. Relationship between $\mathrm{mHAQ}$ for each predominant extremities, and these parameters and sex, age, disease duration of RA, anti-cyclic citrullinated polypeptide antibodies (ACPA), rheumatoid factor (RF), and Sharp/van der Heijde score (SHS), were statistically evaluated using linear regression analysis.

Results: $\mathrm{mHAQ}$ UE significantly correlated with age, ACPA and RF titre, SHS tenderness joint count (TJC), patient's global assessment (PGA), evaluator's global assessment (EGA), C-reactive protein (CRP), US, UL, LL, and PS-vas, whereas $\mathrm{mHAQ}$-LE significantly correlated with all parameters that demonstrated significant correlation with $\mathrm{mHAQ}-\mathrm{UE}$ and disease duration. $\mathrm{mHAQ}$ also correlated with all parameters those that demonstrated significant correlation with $\mathrm{mHAQ}$-LE. Interestingly, all of $\mathrm{mHAQ}-\mathrm{UE}, \mathrm{mHAQ}-\mathrm{LE}$, and $\mathrm{mHAQ}$ did not correlated significantly with swollen joint count (SJC) and LS.

Conclusion: $\mathrm{mHAQ}$ is influenced by various factors, however, SJC and involvement of small joint in lower extremities did not affect $\mathrm{mHAQ}$.

Disclosure of Interests: None declared

DOI: 10.1136/annrheumdis-2021-eular.3644

\begin{tabular}{|l|l|}
\hline POS0575 & THE PATIENT GLOBAL ASSESSMENT (PGA) \\
OF DISEASE ACTIVITY COLLECTS DIFFERENT \\
INFORMATION IN EARLY COMPARED TO \\
ESTABLISHED RHEUMATOID ARTHRITIS
\end{tabular}

L. De Stefano $^{1,2}$, S. Bugatti ${ }^{1,2}$, B. D'Onofrio ${ }^{1,2}$, E. Favalli $^{3}$, A. Manzo ${ }^{1,2}$, R. Caporali ${ }^{3,4}$, C. Montecucco ${ }^{1,2}$. ${ }^{1}$ Fondazione I.R.C.C.S. Policlinico San Matteo, Division of Rheumatology, Pavia, Italy; ${ }^{2}$ The University of Pavia, Department of Internal Medicine and Therapeutics, Pavia, Italy; ${ }^{3}$ ASST Pini-CTO - Presidio Gaetano Pini, Department of Rheumatology, Milano, Italy; ${ }^{4}$ University of Milan, Department of Clinical Sciences \&amp; Community Health, Milano, Italy

Background: The patient global assessment (PGA) is the most widely used patient-reported measure in rheumatoid arthritis (RA), being a component of disease activity scores and definitions of remission. Studies have however shown that, at least in established RA, PGA is mostly associated with pain, functiona limitation, psychological distress and comorbidities, rather than with objective measures of inflammation. As such, the inclusion of PGA as a driver of intensification of immunosuppressive therapy is been questioned. Determinants of PGA may however differ in the earliest stages of RA, when pain processing mechanisms and damage accrual have not occurred yet. Whether the association of PGA with disease activity in RA changes over time is at present undetermined. Objectives: To analyze the associations between PGA and disease activity measures in patients with RA across different phases of the disease.

Methods: 1.002 RA patients from two independent cohorts were included: 1 ) a prospective longitudinal cohort of early RA ( $<12$ months of symptoms) $(n=601)$ with an observation period of 24 months upon initiation of therapy with methotrexate; 2 ) a cross-sectional cohort of established RA (duration $>5$ years) $(n=401)$ with inadequate response to methotrexate. Determinants of PGA were assessed by Pearson's correlation coefficients and multivariable linear regression.

Results: In early RA, median (IQR) symptom duration at inclusion was 15 (9-27) weeks, $71.5 \%$ of the patients were female, $49.3 \%$ were autoantibody-positive, and $30 \%$ had radiographic evidence of $\geq 1$ erosion. The mean (SD) DAS28 was 4.94 (1.19), and the mean (SD) PGA 57.6 (26.6). The proportion of patients at least in low disease activity (DAS28<3.2) after 6, 12 and 24 months of treatment was $49.9 \%, 57.4 \%$ and $67 \%$ respectively. The associations of PGA with pain and functional limitation were moderate to good ( $>0.40$ ) at all time points, and were independent of other co-variates (Table 1) Swollen joints (SJC28) were independently and directly associated with PGA at all time points until 12 months, whilst the association become indirect a 24 months (Table 1). In ACPA-positive patients, PGA at 6 and 12 months was weakly but still significantly associated with SJC28 even in low disease activity states (adj r 0.15 and $0.13, p<0.05$ ). Patients with established RA had a mean [SD] DAS28 of 5.3 [1.2] and were ACPA-positive in $69.6 \%$ of the cases. Independent determinants of PGA were pain, $\mathrm{HAQ}$ and tender joints, whilst no associations with SJC28 and acute phase reactants were found neither in the overall cohort nor in ACPA-positive patients.

Conclusion: In established RA, PGA appears mostly related to factors outside the core domains of disease activity. In contrast, in the early phases of the disease, PGA may more strictly collect information on inflammatory-related symptoms. Better understanding of the relationship between patient reported outcomes and disease activity in the various phases of RA may thus be needed before introducing definitive changes in the current definitions of disease activity. 